\title{
Quantification of Dosimetric Effects of Dental Metallic Implant on Volumetric-Modulated Arc Therapy Plans
}

\author{
Chun-I Lin ${ }^{1}$, Yuanming Feng ${ }^{1 *}$, Wenhuan Jiang ${ }^{1}$ and Zhibin Huang ${ }^{1,2}$
}

${ }^{1}$ East Carolina University, USA

${ }^{2}$ Saint Thomas Rutherford Hospital, USA

*Corresponding author: Yuanming Feng, Ph.D. DABR, Associate Professor, Department of Radiation Oncology, East Carolina University, 600 Moye Blvd, Greenville, NC 27834, USA, Tel: 252-744-8472, E-mail: fengyu@ecu.edu

\begin{abstract}
CT images with the presence of metallic implants may cause severe metal artifacts with incorrect CT numbers, which lead to inaccurate dose calculation in RT planning. The goal of this study was to evaluate the dosimetric impact of metallic implants that correlates with the size of targets and metallic implants and the distance between the two on volumetric-modulated arc therapy (VMAT) plans for head and neck $(\mathrm{H} \& \mathrm{~N})$ cancer patients with dental implants. CT images of six cases were used. Target volumes with different sizes and locations were outlined. In addition, artifact regions excluding surrounding critical organs were contoured and assigned with CT numbers close to water. VMAT plans with half-arc, one-arc and two-arc were generated and same plans were applied to structure sets with and without CT number assignment and compared by $3 \mathrm{D}$ gamma index with the criteria of $2 \%$ of dose-difference and $2 \mathrm{~mm}$ of distance-to-agreement $\left(\gamma_{<2 \% / 2 \mathrm{~mm}}\right)$, dose-volume parameters, conformity index and homogeneity index. The target size showed a vague dosimetric correlation if the target location was far from the implants, however, if the distance between target and implants was close, higher dose discrepancy was found in larger target size. As the target location relative to implants was larger, the $Y_{<2 \% / 2 \mathrm{~mm}}$ passing rate became higher. The larger the implant size, the greater the dose difference would be. Furthermore, half-arc plans demonstrated the greatest impact while two-arc had the smallest or similar influence with one-arc plans. In conclusion, small target size and large high-Z metallic implants with short distance in between can cause severe dosimetric impact.
\end{abstract}

\section{Keywords}

Metal-artifacts, VMAT, Gamma index

\section{Introduction}

In radiation therapy, computed tomography (CT) images are used for target and organ at risk (OAR) delineation and provide electron density information for dose calculation in radiotherapy treatment planning. Accurate $\mathrm{CT}$ data assures the accuracy of dose calculation. However, the presence of metallic objects of high atomic number (high-Z) materials in CT images may create severe metal artifacts accompanied by bright and dark streaking effect due to beam hardening and scattering, which results in incorrect CT numbers in the areas around the metal and jeopardizes the accuracy of the calculated dose to be delivered to the patient.

Therefore, various metal artifact reduction (MAR) methods have been proposed in order to eliminate the metal artifact by either replacing corrupted data or correcting them [1]. The most common approach is to ignore projections affected by the metal objects in the raw data which are corrupted, and apply for different interpolation methods such as linear, polynomial, cubic, spline or wavelet interpolation for recovering the missing data [2-6]. Wei, et al. [4] presented the metal artifact suppression (MAS) method by using smoothing and polynomial interpolation on the projection profile to suppress the artifacts. Meyer, et al. [6] proposed the normalized metal artifact reduction (NMAR) method to improve standard MAR by applying the normalization method for preventing newly introduced artifacts created from interpolation. Meyer, et al. [1] then presented the frequency split metal artifact reduction (FSMAR)

Citation: Chun-I L, Feng Y, Jiang W, Huang Z (2018) Quantification of Dosimetric Effects of Dental Metallic Implant on Volumetric-Modulated Arc Therapy Plans. Int J Cancer Clin Res 5:092. doi. org/10.23937/2378-3419/1410092

Accepted: May 28, 2018; Published: May 30, 2018

Copyright: (C) 2018 Chun-I L, et al. This is an open-access article distributed under the terms of the Creative Commons Attribution License, which permits unrestricted use, distribution, and reproduction in any medium, provided the original author and source are credited. 
method, an inpainting-based method, which combines the high frequencies of an uncorrected image, where all available data were used for the reconstruction with the more reliable low frequencies of an image which was corrected with the NMAR method. Veldkamp, et al. [3] proposed a segmentation by using a Markov random field model (MRF) and interpolation techniques in sinogram for MAS in CT. Abdoli, et al. [7] evaluated five MAR methods, including one-dimensional linear interpolation (LI), two-dimensional interpolation (2D), a NMAR technique, a metal deletion technique, and a maximum a posteriori completion (MAPC) approach for CT images of patients with hip prostheses by using simulation and clinical data. The results showed that simulation studies revealed 2D, NMAR, and MAPC techniques performed almost equally well except for $\mathrm{LI}$ and the visual assessment of clinical data revealed the superiority of NMAR and MAPC in the evaluated pelvic organs and overall image quality.

Furthermore, a number of articles investigated the dosimetirc impact of metallic artifacts with different MAR methods. Wei, et al. [8] proposed that the target may reveal severe underdose if the artifact-contaminated CT image is used instead of the artifact-suppressed one for all proton, electron or photon treatment modalities. Kim, et al. [9] discussed that the presence of metal artifacts can cause relatively hot spots in OARs and cold spots in targets. Li, et al. [10] concluded that their MAR reduced the $\mathrm{CT}$ artifact and improved the $\mathrm{CT}$ number accuracy but there was no significant impact of the calculated dose distributions. Lin, et al. [11] investigated the dosimetric impact of dental implants of three different implant materials: Titanium, Degubond 4 and gold. They found that the main effect of the dental implant was the severe attenuation in the downstream and the planning target volume (PTV) coverage for volumetric modulated arc therapy (VMAT) plans degrades significantly but within clinically acceptable level. Maerz, et al. [12] investigated the dose impact on intensity modulated radiotherapy (IMRT) and VMAT plans with phantoms that consist of homogeneous water equivalent material surrounding dental implants for the accuracy of dose calculations, compared to measured data using EBT2 films and the benefit of MAR using bulk density correction.
The results showed that the accuracy of dose calculations is significantly high on corrected CT images, and the VMAT plan has higher agreement of calculated and measured dose distribution than the IMRT plan. Kamomae, et al. [13] also investigated the dosimetric impact of dental metallic crown (DMC) and the results showed that the dose enhancement and reduction could be mitigated by use of multiple beams and wide gantry rotation angles and for IMRT and VMAT, the dose impact occurred randomly due to the complex combination of beam penetrating the DMC. Spadea, et al. [14] focused on the severity of dosimetric impact for low-Z and high-Z metal implants and found low-Z material had no significant dose discrepancies between CT sets with and without applying MAR algorithm while high-Z implant caused relevant underdose around the metal and overdose downstream of the hardware.

VMAT is an advanced IMRT technology that delivers dose with continuous motion of multi-leaf collimator (MLC) and intensity modulation during $360^{\circ}$ gantry rotation in a single or multi-arc treatment within a few minutes. VMAT improves dose conformity to the target while shortening the treatment period. Previous research showed that VMAT provides similar or even better treatment plan achievement as IMRT [15-17] and possesses higher dose calculation accuracy than IMRT [12].

Even though there are thriving literatures discussing the dosimetric discrepancy of metal implants between corrected and uncorrected CT image sets by MAR methods, seldom mention the effect of metal artifacts pertaining to target and metallic implant sizes and the location of target with metal implants that have been taken into account. The goal of this study was to evaluate the dosimetric impact of metallic implants that correlates with the size of targets and the implants and distance in between on VMAT plans for Head and Neck (H\&N) cancer patients with dental metallic implants.

\section{Materials and Methods}

\section{Patient data and contouring}

CT image data of six $\mathrm{H} \& \mathrm{~N}$ cancer patients with dental metallic implants were used in this study. The volumes of metal implants for these six patients were summa-

Table 1: Summary of the volumes of metal implant and target and distance between target and metallic implant for six head and neck cancer patients.

\begin{tabular}{|c|c|c|c|c|c|c|c|c|}
\hline & Patient 1 & Patient 2 & Patient 3 & Patient 4 & \multicolumn{2}{|c|}{ Patient 5} & \multicolumn{2}{|c|}{ Patient 6} \\
\hline Metal Volume $\left(\mathrm{cm}^{3}\right)$ & 4.8 & 2.6 & 0.6 & 2.9 & \multicolumn{2}{|l|}{4.6} & \multicolumn{2}{|l|}{0.9} \\
\hline \multirow{4}{*}{ Target Volume $\left(\mathrm{cm}^{3}\right)$} & 160.80 & 82.46 & 85.09 & 55.08 & \multicolumn{2}{|l|}{66.77} & \multicolumn{2}{|c|}{63.40} \\
\hline & 121.50 & 60.13 & 49.41 & 37.45 & \multicolumn{2}{|c|}{$51.77^{*}$} & \multicolumn{2}{|c|}{$45.65^{*}$} \\
\hline & 39.10 & $27.40^{*}$ & $29.50^{*}$ & $18.50^{*}$ & \multicolumn{2}{|l|}{$24.12^{*}$} & \multicolumn{2}{|c|}{$22.37^{*}$} \\
\hline & $16.90^{*}$ & 17.78 & & & & & & \\
\hline \multirow{4}{*}{ Distance $(\mathrm{cm})$} & 4.81 & 4.63 & 12.65 & 4.07 & 6.66 & 6.16 & 4.25 & 3.94 \\
\hline & 3.31 & 3.76 & 6.50 & 2.92 & 5.38 & 4.89 & 3.54 & 3.38 \\
\hline & 2.03 & 1.87 & 4.29 & 2.11 & 4.37 & 3.74 & 2.85 & 2.07 \\
\hline & 1.06 & & 2.29 & 1.49 & 3.69 & 2.97 & & \\
\hline
\end{tabular}

"Specific target volume for each patient that is used for different distances between target and metal implant. 
rized in Table 1 . The $\mathrm{CT}$ number of metal implants is considered to be higher than $2000 \mathrm{HU}$ that could causes major metal artifact $[1,4]$. Thus, the metal volume was contoured manually based on the criteria and then measured in the Treatment Planning System (TPS). Various metal implant volumes of different patients allowed us to investigate the dosimetric effect due to the size of the implants. In addition, obtaining exact information of the materials of the metal implants for each patient is difficult in this retrospective study. However, the commonly used materials for metal implants are titanium alloy, titanium, crown, and amalgam with relative electron densities of 3.72, 3.76, 7.17, and 9.09 [18]. The corresponding $\mathrm{HU}$ are all higher than $2000 \mathrm{HU}$. Therefore, contouring the area of CT number higher than $2000 \mathrm{HU}$ to identify the volume of metal implants is reasonable.

Target volumes with different sizes and locations were arbitrarily determined as listed in detail in Table 1 and organ at risk (OARs) such as brain stem, spinal cord, parotid glands, eyes, oral cavity and etc. were also contoured by the same experienced investigator in order to eliminate variations from manual contouring. Metal artifact regions with dark and bright streaks, excluding surrounding critical organs, were outlined and assigned with CT numbers close to water (OHU) which represents soft tissue as water equivalent for regions of inaccurate $\mathrm{CT}$ number. On Table 1, numbers with an asterisk symbol on the row of the target volume indicates the specific target volumes that were utilized to create different distances between target and metallic implants for assessing dosimetric impact under target location consideration.

\section{VMAT planning}

All the VMAT treatment plans in this study were designed using the Eclipse TPS version 11.0.47 and a $6 \mathrm{MV}$ photon beam was used. VMAT plans with half-arc $\left(90^{\circ}\right.$ to $270^{\circ}$ counterclockwise rotation), one-full-arc $\left(181^{\circ}\right.$ $179^{\circ}$ clockwise rotation) and two-full-arcs $\left(181^{\circ}-179^{\circ}\right.$ clockwise and $179^{\circ}-181^{\circ}$ counterclockwise rotations) were constructed with AAA algorithm plan optimization and dose calculation. The prescribed dose of all plans was $69.96 \mathrm{~Gy}(2.12 \mathrm{~Gy} / \mathrm{fr}$. $\times 33$ fraction) and $95 \%$ of PTV was covered by at least $95 \%$ of prescribed dose for all the plans with original CT images. The same plans were applied to structure sets with and without CT number correction of metal artifact regions and the results were compared.

\section{Dose evaluation}

In order to evaluate the dosimetric differences between plans with $\left(\right.$ plan $\left._{\mathrm{HU}}\right)$ and without $\left(\right.$ plan $\left._{\text {ori }}\right)$ replacing CT number of metal artifact regions, the dose matrices of two plans were compared by conducting the following parameters using in-house MATLAB codes. Gamma index [19].

3D gamma index $(\gamma)$ was used to assess and score the dose difference which takes both dose deviation $(\Delta D)$ and Distance-to-agreement (DTA) into account and $\gamma$ is defined as

$$
\gamma=\sqrt{\left(\Delta D / C_{\Delta D}\right)^{2}+\left(D T A / C_{D T A}\right)^{2}}
$$

Where $\Delta D$ compares dose difference of plan ${ }_{\text {ori }}$ and plan $_{\text {Huo }}$ in the same voxel of spatial coordinates; DTA means the shortest distance where two voxels in different plans own the same dose values; $C_{\Delta D}$ is criteria of dose deviation and $C_{D T A}$ is criteria of DTA.

$\Delta \mathrm{D}$ can be written as $\Delta \mathrm{D}=\mathrm{D}_{\text {ori }}\left(r_{\text {ori }}\right)-\mathrm{D}_{\text {HUO }}\left(r_{\text {HUO }}\right)$

Where $D_{\text {ori }}\left(r_{\text {ori }}\right)$ represents the dose in voxel $r_{\text {ori }}$ and $D_{\text {HUO }}\left(r_{\text {HUO }}\right)$ is the dose in voxel $r_{\text {HUO }}$. The disadvantage of dose deviation is that it is very sensitive to small spatial displacements in steep dose gradient regions and the value can be large without actual clinical significance.

DTA can be calculated as DTA $=\min \left\{\left|r_{\text {HUo }^{\prime}} r_{\text {ori }}\right|\right\}$

That the voxels in plan ${ }_{\text {Huo }}$ with dose equal to the dose in $r_{\text {ori }}$ are donated by $r_{\mathrm{Hu}^{\prime}}$. The drawback of DTA is that if the dose in the region surrounding the pixel is approximately homogeneous, the DTA value may become unreasonably large even though the dose difference between $r_{\text {ori }}$ and $r_{\text {HUO }}$ is small.

The criteria of $\psi$ values used in this study were $2 \%$ of $\triangle \mathrm{D}$ and $2 \mathrm{~mm}$ of DTA $\left(\gamma_{<2 \% / 2 \mathrm{~mm}}\right)$ for the dose coverage difference within the $50 \%$ and $90 \%$ isodose volume and also the volume extended $1 \mathrm{~cm}$ outside the metal implants.

\section{Dose-volume parameter}

In terms of the DVH curves of the PTV, dose-volume parameters of the dose received by $95 \%$ PTV $\left(D_{95 \%}\right)$ and the percent volume of the PTV that receives $95 \%$ or more of the prescribed dose $\left(\mathrm{V}_{95 \%}\right)$ was analyzed for evaluation of the PTV dose coverage.

\section{Conformity index \& Homogeneity index}

Conformity index $(\mathrm{Cl})$ determines the quality of the volume of dose distribution that conforms to the target volume. Paddick $\mathrm{Cl}$ [20] was used as

$$
\mathrm{Cl}=\left(\mathrm{TV}_{\mathrm{PIV}}\right)^{2} /(\mathrm{TV} \times \mathrm{PIV})
$$

Where $\mathrm{TV}_{\mathrm{PIV}}$ is the target volume covered by prescription isodose volume, TV is the target volume and PIV is the prescription isodose volume.

Homogeneity Index (HI) [20] is an analysis of the uniformity of dose distribution in the target volume. The $\mathrm{HI}$ was calculated as

$$
H I=\left(D_{1}-D_{99}\right) / D_{p} \times 100
$$

Where $D_{1}$ is the minimum dose to $1 \%$ of the target volume, indicating the "maximum dose"; $\mathrm{D}_{99}$ is the minimum dose to the $99 \%$ of the target volume, indicating the "minimum dose" and $D_{p}$ is the prescribed dose.

The differences of $\mathrm{Cl}(\Delta \mathrm{Cl})$ and $\mathrm{HI}(\Delta \mathrm{HI})$ for dose dis- 
Table 2: Percentage of $\gamma_{<2 \% / 2 \mathrm{~mm}}$ within the $50 \%$ and $90 \%$ isodose volume regarding target size for one-arc, half-arc and two-arc VMAT plans of six patients.

\begin{tabular}{|c|c|c|c|c|c|c|c|}
\hline \multirow{3}{*}{$\begin{array}{l}\text { Patient } \\
\text { [metal implant size }\left(\mathrm{cm}^{3}\right) \text { ] }\end{array}$} & \multirow{3}{*}{ Target size $\left(\mathrm{cm}^{3}\right)$} & \\
\hline & & \multicolumn{2}{|c|}{ one-arc } & \multicolumn{2}{|c|}{ half-arc } & \multicolumn{2}{|c|}{ two-arc } \\
\hline & & $50 \%$ & $90 \%$ & $50 \%$ & $90 \%$ & $50 \%$ & $90 \%$ \\
\hline \multirow{4}{*}{$\begin{array}{l}\text { Patient } 1 \\
{[4.8]}\end{array}$} & 160.80 & 99.13 & 99.17 & 97.75 & 97.63 & 99.33 & 99.37 \\
\hline & 121.50 & 99.73 & 97.73 & 98.78 & 98.28 & 98.79 & 98.59 \\
\hline & 39.10 & 99.71 & 97.34 & 98.55 & 97.10 & 98.88 & 98.73 \\
\hline & 16.90 & 99.26 & 90.72 & 96.07 & 84.80 & 97.53 & 93.87 \\
\hline \multirow{4}{*}{$\begin{array}{l}\text { Patient } 2 \\
{[2.6]}\end{array}$} & 82.46 & 97.95 & 96.25 & 97.48 & 93.93 & 99.09 & 98.75 \\
\hline & 60.13 & 98.34 & 97.18 & 98.33 & 96.79 & 98.83 & 98.04 \\
\hline & 27.40 & 98.26 & 96.32 & 97.10 & 92.73 & 98.71 & 97.52 \\
\hline & 17.78 & 97.60 & 94.66 & 97.31 & 94.08 & 98.67 & 97.38 \\
\hline \multirow{3}{*}{$\begin{array}{l}\text { Patient } 3 \\
{[0.6]}\end{array}$} & 85.09 & 99.27 & 99.56 & 99.14 & 99.36 & 99.26 & 99.56 \\
\hline & 49.41 & 99.28 & 99.36 & 99.32 & 99.21 & 99.44 & 99.56 \\
\hline & 29.50 & 99.48 & 99.60 & 99.18 & 99.25 & 99.32 & 99.39 \\
\hline \multirow{3}{*}{$\begin{array}{l}\text { Patient } 4 \\
{[2.9]}\end{array}$} & 55.08 & 99.11 & 98.98 & 99.28 & 99.23 & 99.40 & 99.46 \\
\hline & 37.45 & 98.65 & 97.83 & 98.42 & 97.05 & 99.25 & 99.09 \\
\hline & 18.50 & 98.89 & 98.59 & 98.70 & 97.46 & 99.14 & 98.97 \\
\hline \multirow{3}{*}{$\begin{array}{l}\text { Patient } 5 \\
{[4.6]}\end{array}$} & 66.77 & 96.93 & 95.75 & 94.18 & 88.60 & 97.64 & 97.27 \\
\hline & 51.77 & 97.07 & 95.84 & 94.98 & 91.19 & 97.30 & 96.54 \\
\hline & 24.12 & 96.74 & 94.54 & 96.92 & 94.14 & 97.57 & 97.57 \\
\hline \multirow{3}{*}{$\begin{array}{l}\text { Patient } 6 \\
{[0.9]}\end{array}$} & 63.40 & 97.78 & 97.56 & 97.59 & 97.45 & 99.10 & 99.17 \\
\hline & 45.65 & 98.62 & 97.92 & 97.33 & 98.19 & 99.07 & 99.06 \\
\hline & 22.37 & 98.13 & 96.98 & 97.41 & 96.21 & 98.99 & 99.07 \\
\hline
\end{tabular}

Table 3: Percentage of $\gamma_{<2 \% / 2 \mathrm{~mm}}$ within the volume extended $1 \mathrm{~cm}$ outside the metal implants regarding target size for one-arc, half-arc and two-arc VMAT plans of six patients.

\begin{tabular}{|c|c|c|c|c|}
\hline \multirow{2}{*}{$\begin{array}{l}\text { Patient } \\
\left.\text { [metal implant size }\left(\mathrm{cm}^{3}\right)\right]\end{array}$} & \multirow{2}{*}{ Target size $\left(\mathrm{cm}^{3}\right)$} & \multicolumn{3}{|l|}{$\gamma_{<2 \% / 2 \mathrm{~mm}}$} \\
\hline & & one-arc & half-arc & two-arc \\
\hline \multirow{4}{*}{$\begin{array}{l}\text { Patient } 1 \\
\text { [4.8] }\end{array}$} & 160.80 & 98.69 & 94.21 & 98.76 \\
\hline & 121.50 & 99.78 & 99.24 & 99.71 \\
\hline & 39.10 & 99.76 & 98.32 & 99.74 \\
\hline & 16.90 & 99.52 & 98.54 & 99.69 \\
\hline \multirow{4}{*}{$\begin{array}{l}\text { Patient } 2 \\
{[2.6]}\end{array}$} & 82.46 & 98.63 & 97.64 & 99.42 \\
\hline & 60.13 & 99.04 & 98.96 & 99.44 \\
\hline & 27.40 & 98.98 & 98.82 & 99.23 \\
\hline & 17.78 & 99.13 & 98.60 & 99.17 \\
\hline \multirow{3}{*}{$\begin{array}{l}\text { Patient } 3 \\
{[0.6]}\end{array}$} & 85.09 & 99.76 & 99.20 & 99.88 \\
\hline & 49.41 & 99.81 & 99.19 & 99.85 \\
\hline & 29.50 & 99.84 & 99.37 & 99.91 \\
\hline \multirow{3}{*}{$\begin{array}{l}\text { Patient } 4 \\
{[2.9]}\end{array}$} & 55.08 & 99.36 & 99.27 & 99.56 \\
\hline & 37.45 & 99.25 & 99.03 & 99.33 \\
\hline & 18.50 & 99.20 & 99.07 & 99.44 \\
\hline \multirow{3}{*}{$\begin{array}{l}\text { Patient } 5 \\
\text { [4.6] }\end{array}$} & 66.77 & 98.65 & 97.13 & 98.32 \\
\hline & 51.77 & 98.22 & 96.66 & 98.44 \\
\hline & 24.12 & 97.59 & 97.01 & 97.90 \\
\hline \multirow{3}{*}{$\begin{array}{l}\text { Patient } 6 \\
{[0.9]}\end{array}$} & 63.40 & 97.40 & 96.37 & 98.57 \\
\hline & 45.65 & 98.28 & 95.83 & 98.71 \\
\hline & 22.37 & 98.57 & 97.83 & 98.26 \\
\hline
\end{tabular}

tributions between plans with and without CT number correction were analyzed in this study.

\section{Results}

\section{Target size}

Table 2 shows the percentage of gamma index with the criteria of $2 \% \Delta \mathrm{D}$ and $2 \mathrm{~mm}$ DTA $\left(\gamma_{<2 \% / 2 \mathrm{~mm}}\right)$ within the $50 \%$ and $90 \%$ isodose volume corresponding to various target sizes for six patients with half-arc, one-full-arc and two-full-arcs plans. In general, if the target location was close enough to the metal implants, lower $\gamma_{<2 \% / 2 \mathrm{~mm}}$ was found in the case of larger target size due to larger overlapping with the CT number replacement region, while smaller target size resulted in higher $\gamma_{<2 \% / 2 \mathrm{~mm}}$. With the target location far from the metal implant, there showed less dosimetric effect for different target sizes. In fact, it is challenging to consider target size only due to the difficulty of contouring different target volumes with the same location of center of mass. The percentage 


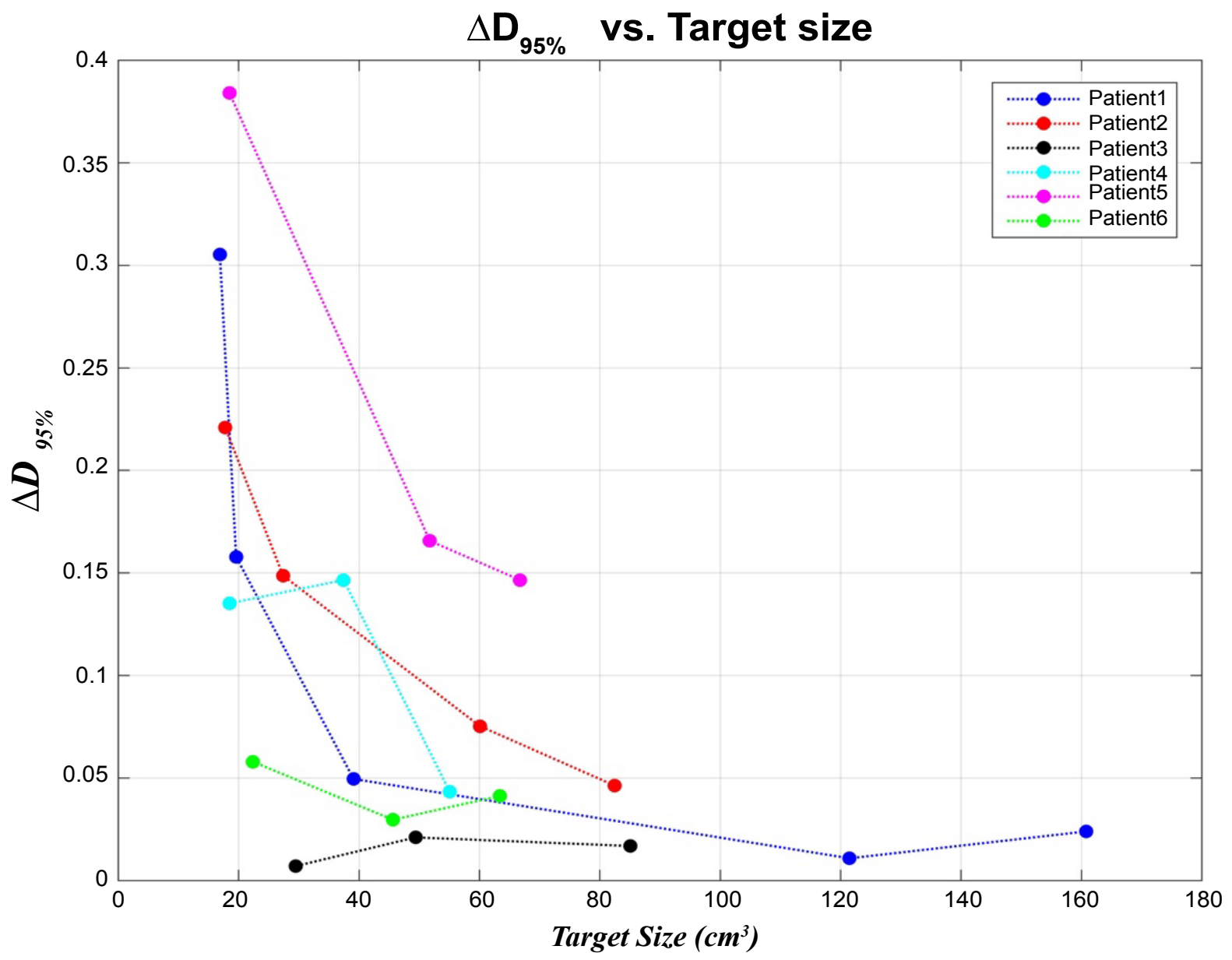

Figure 1: Comparison of the difference of PTV dose coverage $\left(\Delta D_{95 \%}\right)$ between plans with and without CT number correction in regard to target sizes for six patients.

of $V_{<2 \% / 2 \mathrm{~mm}}$ within the region extended $1 \mathrm{~cm}$ from the metal implant is demonstrated in Table 3. This region is considered as the most affected area by metal artifacts surrounding the metal implant. The result presented similar dosimetric effect, as in Table 2, that the smaller target size led to higher passing rate. In addition, half-arc plans always demonstrated greatest impact while twoarc plans had smallest or similar influence with one-arc plans.

As for the $\Delta \mathrm{D}_{95 \%}$ and $\Delta \mathrm{V}_{95 \%}$, the values basically possess the reduced trend with larger target volume as shown in Figure 1 and $\Delta \mathrm{Cl}$ and $\Delta \mathrm{HI}$ had almost no difference $C_{\Delta D}$ All were smaller than 0.1 for all half-arc, one-arc and two-arc VMAT plans.

\section{Distance between target and metallic implants}

With regard to the target location, Table 4 and Table 5 analyze the percentage $\gamma_{<2 \% / 2 \mathrm{~mm}}$ within the regions of $50 \%$ and $90 \%$ isodose and the region extended $1 \mathrm{~cm}$ outside the volume of the metal implants, respectively.

Both results illustrated that the dose discrepancy between plans with and without CT number assignment increased with reducing distance between target and metallic implants for all six patients, i.e. the passing rate of $\psi_{<2 \% / 2 \mathrm{~mm}}$ was higher for larger distance than for shorter distance apart from target to metal, as shown in Figure 2.

Even though some values seemed against the rising tendency of dose difference with decreasing distance between target and metal implants for $\psi_{<2 \% / 2 \mathrm{~mm}}$ values, the dosimetric impact evidently followed the trend if performing the gamma index with the criteria of $1 \% \Delta \mathrm{D}$ and $1 \mathrm{~mm}$ DTA $\left(\gamma_{<1 \% / 1 \mathrm{~mm}}\right)$ since some data falls between 1-2\% $\Delta \mathrm{D}$ and 1-2 mm DTA. Furthermore, comparing three designs of VMAT plans, the half-arc plan revealed the largest difference while two-arc plans owned the smallest variation.

In addition, when considering the $\Delta \mathrm{D}_{95 \%}$, almost all values showed less than 0.5 difference and the $\Delta \mathrm{V}_{95 \%}$ values were near zero for all plans with and without replacing CT number. Moreover, half-arc plans again showed greater impact than one-arc and two-arc plans. The $\Delta \mathrm{Cl}$ and $\Delta \mathrm{HI}$ also demonstrated no significant difference with all values less than 0.1 for all VMAT plans.

\section{Size of metallic implants}

In order to take the size of metallic implants of different patients into account nearly independently, six 


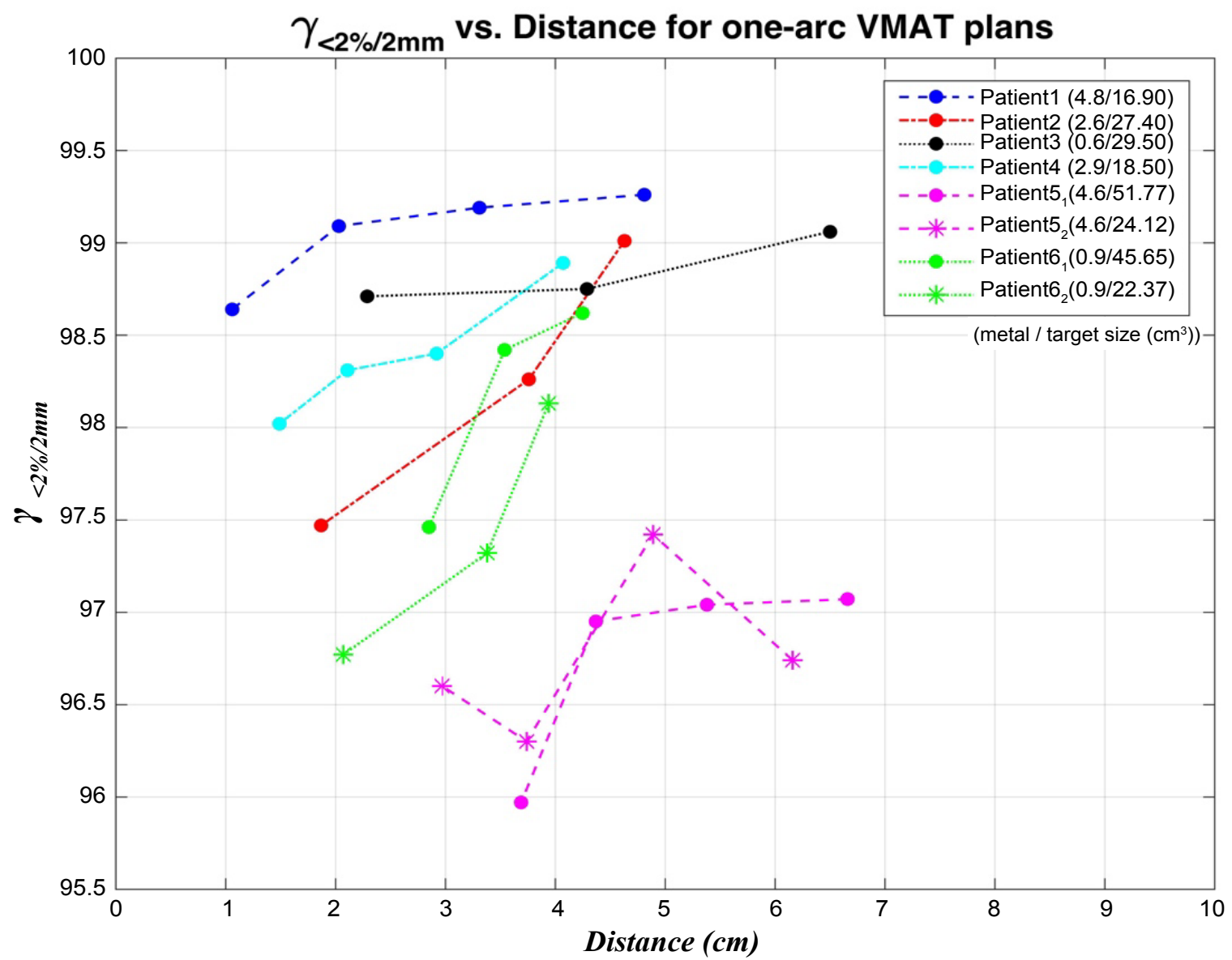

Figure 2: Comparison of the percentage of gamma index with criteria of $2 \% \Delta \mathrm{D}$ and $2 \mathrm{~mm} \mathrm{DTA}\left(\mathrm{Y}_{<2 \% / 2 \mathrm{~mm}}\right)$ of dose distribution between one-arc plans with and without CT number correction within the $50 \%$ isodose volume regarding the distance between target and metallic implants for six patients.

Table 4: Percentage of $\gamma_{<2 \% / 2 \mathrm{~mm}}$ within the $50 \%$ and $90 \%$ isodose volume regarding distance between target and metallic implants for one-arc, half-arc and two-arc VMAT plans of six patients.

\begin{tabular}{|c|c|c|c|c|c|c|c|}
\hline \multirow{3}{*}{$\begin{array}{l}\text { Patient } \\
\left.\text { (target size }\left(\mathrm{cm}^{3}\right)\right) \\
{\left[\text { metal implant size }\left(\mathrm{cm}^{3}\right)\right]}\end{array}$} & \multirow{3}{*}{ Distance $(\mathrm{cm})$} & \multicolumn{6}{|c|}{$\gamma_{<2 \% / 2 \mathrm{~mm}}$} \\
\hline & & \multicolumn{2}{|c|}{ one-arc } & \multicolumn{2}{|c|}{ half-arc } & \multicolumn{2}{|c|}{ two-arc } \\
\hline & & $50 \%$ & $90 \%$ & $50 \%$ & $90 \%$ & $50 \%$ & $90 \%$ \\
\hline \multirow{4}{*}{$\begin{array}{l}\text { Patient } 1 \\
(16.90) \\
{[4.8]}\end{array}$} & 4.81 & 99.26 & 90.72 & 96.07 & 84.80 & 97.53 & 93.87 \\
\hline & 3.31 & 99.19 & 90.92 & 94.72 & 79.48 & 95.84 & 89.07 \\
\hline & 2.03 & 99.09 & 89.76 & 89.92 & 75.81 & 92.83 & 82.90 \\
\hline & 1.06 & 98.64 & 84.37 & 87.11 & 74.45 & 93.46 & 80.38 \\
\hline \multirow{3}{*}{$\begin{array}{l}\text { Patient } 2 \\
(27.40) \\
{[2.6]}\end{array}$} & 4.63 & 99.01 & 98.29 & 97.56 & 93.82 & 98.97 & 98.18 \\
\hline & 3.76 & 98.26 & 96.32 & 97.10 & 92.73 & 98.71 & 97.52 \\
\hline & 1.87 & 97.47 & 94.61 & 97.44 & 91.68 & 98.52 & 96.84 \\
\hline \multirow{4}{*}{$\begin{array}{l}\text { Patient } 3 \\
(29.50) \\
{[0.6]}\end{array}$} & 12.65 & 99.48 & 99.60 & 99.18 & 99.25 & 99.32 & 99.39 \\
\hline & 6.50 & 99.06 & 99.26 & 98.89 & 98.48 & 99.20 & 99.39 \\
\hline & 4.29 & 98.75 & 97.92 & 98.68 & 97.57 & 99.26 & 99.30 \\
\hline & 2.29 & 98.71 & 98.14 & 98.07 & 96.99 & 99.18 & 99.10 \\
\hline \multirow{4}{*}{$\begin{array}{l}\text { Patient } 4 \\
(18.50) \\
{[2.9]}\end{array}$} & 4.07 & 98.89 & 98.59 & 98.70 & 97.46 & 99.14 & 98.97 \\
\hline & 2.92 & 98.40 & 96.94 & 97.95 & 96.17 & 98.79 & 98.27 \\
\hline & 2.11 & 98.31 & 97.09 & 97.08 & 94.18 & 98.85 & 98.32 \\
\hline & 1.49 & 98.02 & 96.76 & 95.45 & 90.21 & 98.60 & 97.59 \\
\hline \multirow{4}{*}{$\begin{array}{l}\text { Patient } 5 \\
(51.77) \\
{[4.6]}\end{array}$} & 6.66 & 97.07 & 95.84 & 94.98 & 91.19 & 97.30 & 96.54 \\
\hline & 5.38 & 97.04 & 95.90 & 95.24 & 92.27 & 96.66 & 94.82 \\
\hline & 4.37 & 96.95 & 95.88 & 95.41 & 91.99 & 96.89 & 95.59 \\
\hline & 3.69 & 95.97 & 93.26 & 94.45 & 91.50 & 96.70 & 94.86 \\
\hline \multirow{4}{*}{$\begin{array}{l}(24.12) \\
{[4.6]}\end{array}$} & 6.16 & 96.74 & 94.54 & 96.92 & 94.14 & 97.57 & 97.57 \\
\hline & 4.89 & 97.42 & 97.84 & 96.01 & 93.55 & 97.03 & 95.26 \\
\hline & 3.74 & 96.30 & 94.91 & 96.03 & 93.97 & 96.84 & 94.32 \\
\hline & 2.97 & 96.60 & 96.17 & 93.63 & 90.00 & 96.69 & 92.66 \\
\hline
\end{tabular}




\begin{tabular}{|l|l|l|l|l|l|l|l|}
\hline $\begin{array}{l}\text { Patient 6 } \\
(45.65)\end{array}$ & 4.25 & 98.62 & 97.92 & 97.33 & 98.19 & 99.07 & 99.06 \\
\hline$[0.9]$ & 3.54 & 98.42 & 98.05 & 96.72 & 96.05 & 98.97 & 98.95 \\
\hline & 2.85 & 97.46 & 97.19 & 96.17 & 96.80 & 98.22 & 98.85 \\
\hline$(22.37)$ & 3.94 & 98.13 & 96.98 & 97.41 & 96.21 & 98.99 & 99.07 \\
{$[0.9]$} & 3.38 & 97.32 & 94.15 & 95.81 & 95.21 & 98.83 & 98.82 \\
\hline
\end{tabular}

Table 5: Percentage of $\gamma_{<2 \% / 2 \mathrm{~mm}}$ within the volume extended $1 \mathrm{~cm}$ outside the metal implants regarding distance between target and metallic implants for one-arc, half-arc and two-arc VMAT plans of six patients.

\begin{tabular}{|c|c|c|c|c|}
\hline \multirow{2}{*}{$\begin{array}{l}\text { Patient } \\
\left.\text { (target size }\left(\mathrm{cm}^{3}\right)\right) \\
\text { [metal implant size } \\
\left.\left(\mathrm{cm}^{3}\right)\right]\end{array}$} & \multirow[b]{2}{*}{$\begin{array}{l}\text { Distance } \\
(\mathrm{cm})\end{array}$} & \multicolumn{3}{|l|}{$\gamma_{<2 \% / 2 \mathrm{~mm}}$} \\
\hline & & $\begin{array}{l}\text { one- } \\
\text { arc }\end{array}$ & $\begin{array}{l}\text { half- } \\
\text { arc }\end{array}$ & $\begin{array}{l}\text { two- } \\
\text { arc }\end{array}$ \\
\hline \multirow{4}{*}{$\begin{array}{l}\text { Patient } 1 \\
(16.90) \\
{[4.8]}\end{array}$} & 4.81 & 99.52 & 98.54 & 99.59 \\
\hline & 3.31 & 98.69 & 97.12 & 99.00 \\
\hline & 2.03 & 97.79 & 94.34 & 97.54 \\
\hline & 1.06 & 96.17 & 92.77 & 96.40 \\
\hline \multirow{3}{*}{$\begin{array}{l}\text { Patient } 2 \\
(27.40) \\
{[2.6]}\end{array}$} & 4.63 & 99.67 & 99.22 & 99.42 \\
\hline & 3.76 & 98.98 & 98.82 & 99.23 \\
\hline & 1.87 & 99.08 & 97.76 & 99.16 \\
\hline \multirow{4}{*}{$\begin{array}{l}\text { Patient } 3 \\
(29.50) \\
{[0.6]}\end{array}$} & 12.65 & 99.84 & 99.37 & 99.91 \\
\hline & 6.50 & 99.58 & 99.34 & 99.52 \\
\hline & 4.29 & 99.51 & 99.42 & 99.56 \\
\hline & 2.29 & 99.06 & 98.98 & 99.14 \\
\hline \multirow{4}{*}{$\begin{array}{l}\text { Patient } 4 \\
(18.50) \\
{[2.9]}\end{array}$} & 4.07 & 99.21 & 99.07 & 99.44 \\
\hline & 2.92 & 98.94 & 98.31 & 98.90 \\
\hline & 2.11 & 98.46 & 97.80 & 98.70 \\
\hline & 1.49 & 98.19 & 96.47 & 98.61 \\
\hline \multirow{4}{*}{$\begin{array}{l}\text { Patient } 5 \\
(51.77) \\
{[4.6]}\end{array}$} & 6.66 & 98.22 & 96.66 & 98.44 \\
\hline & 5.38 & 97.66 & 97.23 & 97.11 \\
\hline & 4.37 & 97.33 & 96.74 & 97.01 \\
\hline & 3.69 & 96.21 & 95.02 & 96.33 \\
\hline \multirow{4}{*}{$\begin{array}{l}(24.12) \\
{[4.6]}\end{array}$} & 6.16 & 97.58 & 97.01 & 97.90 \\
\hline & 4.89 & 97.37 & 97.19 & 97.65 \\
\hline & 3.74 & 96.64 & 95.88 & 97.21 \\
\hline & 2.97 & 96.83 & 95.22 & 96.76 \\
\hline \multirow{4}{*}{$\begin{array}{l}\text { Patient } 6 \\
(45.65) \\
{[0.9]}\end{array}$} & 4.25 & 99.39 & 99.63 & 99.26 \\
\hline & 3.54 & 98.78 & 98.10 & 99.09 \\
\hline & 2.85 & 98.28 & 95.83 & 98.71 \\
\hline & 3.94 & 99.85 & 99.87 & 99.80 \\
\hline \multirow{2}{*}{$\begin{array}{l}(22.37) \\
{[0.9]}\end{array}$} & 3.38 & 98.17 & 98.86 & 98.99 \\
\hline & 2.07 & 98.57 & 97.82 & 98.26 \\
\hline
\end{tabular}

patients were discussed in three main groups by the metal implant size: Patient 1 and patient 5 , with sizes of 4.8 and $4.6 \mathrm{~cm}^{2}$; patient 2 and patient 4 , with sizes of 2.6 and $2.9 \mathrm{~cm}^{2}$; and patient 3 and patient 6 , with sizes smaller than $0.6 \mathrm{~cm}^{2}$ and $0.9 \mathrm{~cm}^{2}$. The patients were separated into several subgroups associated with similar target size and similar distance between target and metallic implants. Consequently, for most cases, the larger the size of the metallic implant, the smaller the $\gamma_{<}$ $2 \% / 2 \mathrm{~mm}$ (Table 4 and Table 5) values would be.

\section{Discussion}

Metallic artifacts in CT images caused by the presence of high- $Z$ and high-density materials contribute to the uncertainty of dose calculation in VMAT planning for H\&N cancer patients with dental metallic implants.
In this study, we focused on investigating the dosimetric impact of metallic implants that are associated with the size of targets and metallic implants as well as distance between the target and the implants on VMAT plans in CT image with and without CT number assignment on the metal artifact region.

The target size showed vague correlation of dosimetric impact because the influential factors not only included target size, but also location information. However, if the target is close enough to the metal implant, smaller target size results in higher $\gamma_{<2 \% / 2 \mathrm{~mm}}$ passing rate. Considering the target location relative to the metallic implants, the closer they are, the larger the dose discrepancies would be. This is because the overlapping region with reassignment of $\mathrm{CT}$ number is larger. On the contrary, the target location related to metal implants with the same target size presented clear relevancy that shorter distances between target and metal implants lead to larger error in dose computation. As for metal implant size, higher passing rate of $\psi_{<2 \% / 2 \mathrm{~mm}}$ was found in smaller size of metal implants. However, both patient 1 and patient 5 had larger metal implants of about 4.7 $\mathrm{cm}^{2}$ but patient 5 displayed larger impact of dose distribution with and without CT number replacement. This might be due to the different material of metal implants which was not taken into account in this study. This was one of the other factors that influenced the dose distribution owing to $\mathrm{CT}$ metal artifacts that was discussed by Spadea, et al. [14], high-Z metal implant caused larger dose differences than the low- $Z$.

Three sets of gamma index analysis with $\Delta \mathrm{D}$ of $2 \%$ and DTA of $2 \mathrm{~mm}\left(\gamma_{<2 \% / 2 \mathrm{~mm}}\right)$ were performed, focusing on three certain regions: Volumes within $50 \%$ and $90 \%$ isodose regions and volume extended $1 \mathrm{~cm}$ from the target instead of the whole involved volumes. The first two scored PTV dose coverage difference of low and high dose region. If the second value is higher than the first one, it indicates that the dose error falls in the lower dose region, and vice versa. The third one evaluated the most affected CT metal artifact region surrounding metallic implants. Other dose evaluation values, $\mathrm{D}_{95 \%}, \mathrm{~V}_{95 \%}$, $\mathrm{Cl}$ and $\mathrm{HI}$, were all small for all the plans. Larger dose impact was showed if the metallic implant was large and close to the target with small target size. Otherwise, the difference was less clinically significant. The dose error for these values was averaged out by taking the whole large involved and uninvolved volume into the calculation of those parameters rather than a specific region that resulted in a small evaluated number. This means $\mathrm{D}_{95 \%}, \mathrm{~V}_{95 \%}, \mathrm{Cl}$ and $\mathrm{HI}$ are not as promising as the gamma 
index assessed within a certain region in considering metal artifact case.

In terms of the design of VMAT plans with half-arc, one-arc and two-arc, the results demonstrated that two-arc plans held highest agreement of the dose distribution than one-arc plans which had similar or slightly larger dose discrepancy. Half-arc plans which showed the largest perturbation of dose distribution between plans with and without CT number correction. More arcs alleviate the dose error caused by metal artifacts because of relatively less contribution to the target dose from beams going through the regions with metallic artifacts. Therefore, half-arc plans had particularly large dose differences due to most of the beam path was delivered from the anterior direction with wide affected region of CT metal artifact.

\section{Conclusion}

Incorrect CT number causes inaccurate calculated dose distribution, therefore, appropriately overwriting metallic artifact regions with reasonable CT numbers or applying metal artifact reduction method to diminish dose calculation error is recommended. Smaller target size and larger high-Z metallic implants with shorter distance in between can cause severer dosimetric impact. Plans with more arcs may alleviate the effect due to relatively less contribution to target dose from the beams passing through the regions with metallic artifacts.

\section{Conflict of Interest}

None.

\section{Funding}

This research did not receive any specific grant from funding agencies in the public, commercial, or not-forprofit sectors.

\section{Declaration of Interest Statement}

The authors do not have conflicts of interest regarding publication of this article.

\section{References}

1. Meyer E, Raupach R, Lell M, Schmidt B, Kachelriess $M$ (2012) Frequency split metal artifact reduction (FSMAR) in computed tomography. Med Phys 39: 1904-1916.

2. Spadea MF, Verburg J, Baroni G, Seco J (2013) Dosimetric assessment of a novel metal artifact reduction method in CT images. J Appl Clin Med Phys 14: 4027.

3. Veldkamp WJ, Joemai RM, van der Molen AJ, Geleijns J (2010) Development and validation of segmentation and interpolation techniques in sinograms for metal artifact suppression in CT. Med Phys 37: 620-628.

4. Wei J, Chen L, Sandison GA, Liang Y, Xu LX (2004) X-ray CT high-density artefact suppression in the presence of bones. Phys Med Biol 49: 5407-5418.

5. Bal M, Spies L (2006) Metal artifact reduction in CT using tissue-class modeling and adaptive prefiltering. Med Phys 33: 2852-2859.
6. Meyer E, Raupach R, Lell M, Schmidt B, Kachelriess M (2010) Normalized metal artifact reduction (NMAR) in computed tomography. Med Phys 37: 5482-5493.

7. Abdoli M, Mehranian A, Ailianou A, Becker M, Zaidi $H$ (2016) Assessment of metal artifact reduction methods in pelvic CT. Med Phys 43: 1588.

8. Wei J, Sandison GA, Hsi WC, Ringor M, Lu X (2006) Dosimetric impact of a CT metal artefact suppression algorithm for proton, electron and photon therapies. Phys Med Biol 51: 5183-5197.

9. Kim Y, Tome WA, Bal M, McNutt TR, Spies L (2006) The impact of dental metal artifacts on head and neck IMRT dose distributions. Radiother Oncol 79: 198-202.

10. Li H, Noel C, Chen H, Harold Li H, Low D, et al. (2012) Clinical evaluation of a commercial orthopedic metal artifact reduction tool for $C T$ simulations in radiation therapy. Med Phys 39: 7507-7517.

11. Lin MH, Li J, Price RA, Wang L, Lee CC, et al. (2013) The dosimetric impact of dental implants on head-and-neck volumetric modulated arc therapy. Phys Med Biol 58: 10271040.

12. Maerz M, Koelbl O, Dobler B (2015) Influence of metallic dental implants and metal artefacts on dose calculation accuracy. Strahlenther Onkol 191: 234-241.

13. Kamomae $T$, Itoh $Y$, Okudaira $K$, Nakaya $T$, Tomida $M$, et al. (2016) Dosimetric impact of dental metallic crown on intensity-modulated radiotherapy and volumetric-modulated arc therapy for head and neck cancer. J Appl Clin Med Phys 17: 234-245.

14. Spadea MF, Verburg JM, Baroni G, Seco J (2014) The impact of low-Z and high-Z metal implants in IMRT: A Monte Carlo study of dose inaccuracies in commercial dose algorithms. Med Phys 41: 011702.

15. Popescu CC, Olivotto IA, Beckham WA, Ansbacher W, Zavgorodni S, et al. (2010) Volumetric modulated arc therapy improves dosimetry and reduces treatment time compared to conventional intensity-modulated radiotherapy for locoregional radiotherapy of left-sided breast cancer and internal mammary nodes. Int J Radiat Oncol Biol Phys 76: 287-295.

16. Vanetti E, Clivio A, Nicoloni G, Fogliata A, Ghosh-Laskar $S$, et al. (2009) Volumetric modulated arc radiotherapy for carcinomas of the oro-pharynx, hypo-pharynx and larynx: A treatment planning comparison with fixed field IMRT. Radiother Oncol 92: 111-117.

17. Anniken D (2013) Comparison of Dose Distributions resulting from IMRT and VMAT, and assessment of MLC leaf positioning errors. Norwegian University, Norway.

18. Catli S (2015) High-density dental implants and radiotherapy planning: Evaluation of effects on dose distribution using pencil beam convolution algorithm and Monte Carlo method. J Appl Clin Med Phys 16: 46-52.

19. Feuvret L, Noel G, Mazeron JJ, Bey P (2006) Conformity index: A review. Int J Radiat Oncol Biol Phys 64: 333-342.

20. Kataria T, Sharma K, Subramani V, Karrthick KP, Bisht SS (2012) Homogeneity Index: An objective tool for assessment of conformal radiation treatments. J Med Phys 37: 207-213.
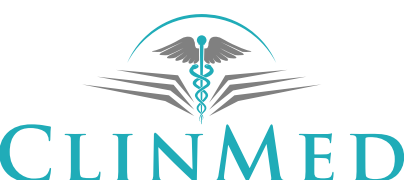

INTERNATIONAL LIBRARY 